\title{
Numerical integration of the contravariant form of the two phase flow motion equations
}

\author{
F. Gallerano \& G. Cannata \\ Department of Hydraulics, University of Rome "La Sapienza", Italy
}

\begin{abstract}
Reservoirs (which are created by the artificial barrage of watercourses) gradually lose the capacity to store water resources owing to the progressive accumulation of solid particles, which are transported by the watercourses, on the bed. In conditions characterized by a flood with a high capacity for transporting large quantities of solid material, turbidity currents could occur; in these situations and in the presence of a steep bed slope, the turbidity currents can reach the bottom outlets. In this paper a model for the simulation of the turbidity currents is proposed; the model is based on the numerical integration of the two phase flow motion equations. These equations are integrated on a three-dimensional generalised curvilinear grid and are directly expressed in contravariant formulation. The numerical procedure adopted is based on a fractional step method and the proposed numerical scheme is conservative and fourth order accurate. The numerical model is validated by comparison between the numerical results and experimental data and is applied to the reservoir of Pieve di Cadore (Italy), in order to verify the possibility of the production of turbidity currents and the possibility of using the bottom outlet to discharge downstream the solid material that reaches the reservoir during flood events.
\end{abstract}

Keywords: two phase flow, turbidity current, contravariant form, reservoir.

\section{Introduction}

Reservoirs (which are created by the artificial barrage of watercourses) gradually lose the capacity to store water resources owing to the progressive accumulation of solid particles, which are transported by the watercourses, on the bed (the silting process). In this paper the possibility of using the bottom outlets to avoid or reduce the solid particle sedimentation (which occurs during flood events) is 
evaluated. Most of the solid particles that accumulate in the reservoirs are transported by the tributary during floods. In conditions characterized by a flood with a high capacity for transporting large quantities of solid material, turbidity currents could occur; in these situations, and in the presence of a steep bed slope, the turbidity currents can reach the bottom outlets. The well timed opening of the bottom outlets can permit the discharge downstream of most of the solid material that is transported by the currents during the flooding. In order to verify the possibility of using the bottom outlets for the optimal control of the silting process in the reservoir, it is necessary to verify the possibility that the turbidity currents occur in the reservoir.

In this paper a model for the simulation of the turbidity currents is proposed; the model is based on the numerical integration of the two phase flow motion equations. These equations are integrated on a three-dimensional generalised curvilinear grid and are directly expressed in contravariant formulation. The numerical model is validated by the comparison between the numerical results and experimental data of Hosseini et al. [1] and is applied to the reservoir of Pieve di Cadore (Italy), in order to verify the possibility of the production of turbidity currents and the possibility of using the bottom outlet to discharge downstream the solid material that reaches the reservoir during flooding.

\section{Contravariant formulation of the two-phase flow motion equations}

The simulation of the turbidity currents in an artificial reservoir requires the numerical integration of motion equations of a two phase flow with a mutual interaction between the phases. In the formalization of the above mentioned equations, some hypotheses can be adopted to simplify the representation of the physical phenomena: a) there is a thermal and dynamic equilibrium between the phases; b) the solid phase can be considered as a homogeneous fluid, so that the mass and momentum conservation principles are valid for it; c) the solid particles are spherical in shape with diameter " $d$ ".

Let $\tilde{\rho}_{s}$ be the microscopic density of the single particle; $\rho_{s}$ the macroscopic density of the solid phase; $a=\rho / \tilde{\rho}_{s}$ the volume fraction of the solid phase; $\rho$ the microscopic density of the fluid phase; $\rho_{f}$ the macroscopic density of the fluid phase. The physical domain (in which the turbidity currents generally occur) are characterised by a high level of morphological complexity. In this paper the definition of a computational domain (which represents the geometry of the reservoir) is realised by a generalised curvilinear grid. The two phase flow motion equations are written directly in the contravariant formulation in a curvilinear coordinate system. In order to introduce the notation to be used, we consider a transformation $x^{l}=x^{l}\left(\xi^{1}, \xi^{2}, \xi^{3}\right)$ from the Cartesian coordinates $\vec{x}$ to the curvilinear coordinates $\vec{\xi}$ (note that superscripts indicate components and not powers in the present notation). Let $\vec{g}_{(l)}=\partial \vec{x} / \partial \xi^{l}$ be the covariant base 
vectors and $\vec{g}^{(l)}=\operatorname{grad}\left(\xi^{l}\right)$ the contravariant base vectors. The metric tensor and its inverse are defined by $g_{l m}=\vec{g}_{(l)} \cdot \vec{g}_{(m)}$ and $g^{l m}=\vec{g}^{(l)} \cdot \vec{g}^{(m)}(l, m=1,3)$. The Jacobian of the transformation is given by $\sqrt{g}=\sqrt{\operatorname{det}\left(g_{l m}\right)}$. The transformation relationship between the fluid phase velocity vector, $\vec{v}$, in the Cartesian coordinate system and its contravariant components, $u^{l}$, in the curvilinear coordinate system are given by $u^{l}=\vec{g}^{(l)} \cdot \vec{v}$ and $\vec{v}=u^{l} \vec{g}_{(l)}$ [2].

The same relationship also applies to other vectors. In the following equations a comma with an index in a subscript denotes covariant differentiation. The covariant derivative is defined as $u^{l},_{m}=\partial u^{l} / \partial \xi^{m}+\Gamma_{m n}^{l} u^{n}$ where $\Gamma_{m n}^{l}$ is the Cristoffel symbol that is given by $\Gamma_{m n}^{l}=\vec{g}^{(l)} \cdot \partial \vec{g}_{(n)} / \partial \xi^{m}[3]$.

For the fluid phase the mass and the momentum balance equations in contravariant form are:

$$
\begin{gathered}
\rho \frac{\partial(1-a)}{\partial t}+\rho\left[(1-a) u^{l}\right],,_{l}=0 \\
\rho \frac{\partial(1-a) u^{l}}{\partial t}+\rho\left[(1-a) u^{l} u^{m}\right],,_{m}=\rho(1-a) f^{l}-T^{l m},{ }_{m}-F_{p}^{l}
\end{gathered}
$$

where $f^{l}$ and $T^{l m}$ are the contravariant components (in the curvilinear coordinate system) of the external body forces and the stress tensor, respectively. The covariant derivative of a second order tensor is given by $T^{l m}{ }_{m}=(1 / \sqrt{g})\left(\partial \sqrt{g} T^{l m} / \partial \xi_{m}\right)+T^{m n} \Gamma_{m n}^{l} . F_{p}^{l}$ is the contravariant component (in the curvilinear coordinate system) of the force per unit volume resulting from the interaction between the two phases, which is given by

$$
F_{p}^{l}=a \frac{18 \mu_{f}}{d^{2}}\left(u^{l}-u_{s}^{l}\right)-a g^{l m} p_{f},_{m}
$$

where $\mu_{f}$ and $p_{f}$ are, respectively, the viscosity and the pressure of the fluid phase and $u_{s}^{l}$ is the contravariant component (in the curvilinear coordinate system) of the solid phase velocity. The first term on the right-hand side of Eq. (3) is the viscous resistance force according to Stokes' law, while the second term is due to the pressure gradient, in the fluid surrounding the particle, caused by the acceleration of the fluid.

The contravariant form of the equations for the solid phase are:

$$
\begin{gathered}
\frac{\partial a \tilde{\rho}_{s}}{\partial t}+\left(a \tilde{\rho}_{s} u_{s}^{l}\right)_{l}=0 \\
\tilde{\rho}_{s} \frac{\partial a u_{s}^{l}}{\partial t}+\tilde{\rho}_{s}\left[a u_{s}^{l} u_{s}^{m}\right],_{m}=a \tilde{\rho}_{s} f^{l}-T_{s}^{l m},_{m}+F_{p}^{l}
\end{gathered}
$$


where $T_{s}^{l m}$ is the contravariant component of the tensor that takes into account the effects of the field interactions between two or more particles (the effect of individual particles being given by the drag force $F_{p}^{l}$ ). When the analysis is restricted to diluted suspension, Equation (5) can be simplified: since $T_{s}^{l m}$ is inversely proportional to the ratio of the distance between particles to their diameter (and is therefore small for diluted suspensions), it can be neglected; furthermore, the volume fraction $a$ can be neglected in the continuity equation and in the material derivative of the velocity in the momentum balance equation of the fluid phase. With these assumptions the equations for the two-phase flow become:

$$
\begin{gathered}
\rho u^{l}{ }_{l}=0 \\
\rho \frac{\partial u^{l}}{\partial t}+\rho u^{l} u^{m}{ }_{m}=\rho(1-a) f^{l}-T^{l m},_{m}-F_{p}^{l} \\
\frac{\partial a \tilde{\rho}_{s}}{\partial t}+\left(a \tilde{\rho}_{s} u_{s}^{l}\right)_{l}=0 \\
\tilde{\rho}_{s} \frac{\partial a u_{s}^{l}}{\partial t}+\tilde{\rho}_{s}\left[a u_{s}^{l} u_{s}^{m}\right],_{m}=a \tilde{\rho}_{s} f^{l}+F_{p}^{l}
\end{gathered}
$$

The two sets of equations, (6)-(7) and (8)-(9), are coupled through the momentum interchange term $F_{p}^{l}$ and the volume fraction $a$.

In order to evaluate the different level of coupling between the sets of equations, generally the loading ratio of the flow, $\beta=\tilde{\rho}_{s} / \rho$, and the Stokes number, $S k=\tau_{p} / \tau_{f}$, are introduced; $\tau_{p}$ is the particle translational relaxation time and $\tau_{f}$ is the characteristic time of fluid flow. The relaxation time can be expressed as $\tau_{p}=\tilde{\rho}_{s} d^{2} /\left(18 \mu_{f} f_{d}\right)$, where the coefficient $f_{d}=1+(1 / 6) \operatorname{Re}_{p}^{2 / 3}$ is the drag coefficient, which is a function of the particle Reynolds number $\operatorname{Re}_{p}=\rho d\left|\vec{v}-\vec{v}_{s}\right| / \mu_{f}$ ( $d$ and $\vec{v}_{s}$ are the diameter and the velocity vector of the solid particle, respectively). The value of $S k$ indicates how rapidly the particle is able to follow the fluid velocity variations: the rapidity of these variations may be represented by the characteristic time of the flow field, $\tau_{f}=L / U$, where $L$ and $U$ are, respectively, the flow field characteristic length and the flow characteristic velocity. The Stokes number $S k$ is proportional to the translational nonequilibrium of the particles. A small value of $S k$ means a small difference between particle and fluid velocities but a large coupling effect.

The loading ratio $\beta$ multiplies the effects of the Stokes number on the momentum equations, increasing the coupling of the fluid with the particle field. For very small values of $\beta$ the fluid phase is independent of the solid phase and only a one way coupling occurs between the two equation systems. The value of the Stokes number indicates whether it is appropriate to consider the particles to be in equilibrium with the fluid flow. For $S k<10^{-1}$ the flow is only slightly out 
of equilibrium, which allows us to assume that the particle velocity field is a superimposing of the fluid field and the particle settling velocity. For $S k>10^{-1}$ the nonequilibrium effects become significant and imply a large relaxation time between the particles and the fluid. For a large value of the loading factor $\beta$ a two-way coupling occurs between the two equation systems and they must be solved simultaneously. The effect of the particles on the fluid flow field cannot be neglected anymore. In the case of turbidity currents small values of $S k$ give us the possibility to consider that the particles are in equilibrium with the fluid flow. Consequently the particle velocity field can be assumed as a superimposing of the fluid flow field and the particle settling velocity. On the other hand, the large values of $\beta$ indicate that the effects of the particles on the fluid field cannot be neglected. Thus for the turbidity currents the hypothesis of the "linearization" can be introduced: the particle velocity field is simply obtained as a superimposing of the flow field and the particle settling velocity, and the particle volume fraction is calculated by solving the particle concentration equation.

For a two phase turbulent flow the independent variables $u^{l}, p, a$ are split into a mean and a fluctuating part, $u^{l}=\bar{u}^{l}+u^{\prime}, p=\bar{p}+p^{\prime}, a=a+a^{\prime}$, in order to take into account the effects of turbulence. The linearised model can be deduced as a particular case of the system (6)-(9) by assuming $w_{\text {sed }}$ for the settling velocity of the particles in a quiescent surrounding fluid. Let us indicate with $\vec{w}_{s}$ the vector whose components in the Cartesian coordinate system are $\left\{0,0, w_{\text {sed }}\right\}$. The contravariant counterpart in the curvilinear coordinate system of $\vec{w}_{s}$ is given by $w^{l}=\vec{g}^{(l)} \cdot \vec{w}_{s}$. With this assumption the equations for the turbulent two phase flow are:

$$
\begin{gathered}
u_{,_{l}{ }_{l}=0} \\
\frac{\partial \bar{u}^{l}}{\partial t}+\bar{u}^{l} \bar{u}^{m},_{m}=(1+R \bar{a}) \bar{f}^{l}-\frac{1}{\rho} g^{m l} \bar{p},_{m}+T_{R}^{l m},_{m} \\
\frac{\partial \bar{a}}{\partial t}+\left(\bar{a} \bar{u}^{l}\right)_{, l}-\left(\bar{a} w^{l}\right){ }_{{ }_{l}}+b^{l},_{l}=0
\end{gathered}
$$

where $T_{R}^{l m}$ are the contravariant components of the turbulent stress tensor, $b^{l}=\overline{u^{l} a^{\prime}}$ and $R=\left(\tilde{\rho}_{s} / \rho\right)-1 . T_{R}^{l m}$ and $b^{l}$ can be related to the kinetic energy of turbulence, $k=\overline{u^{l} u^{l}}$, and to the mean dissipation rate per unit mass of fluid due to the turbulence, $\varepsilon$.

\section{The numerical scheme}

Equations (10), (11) and (12) are integrated, in the computational domain, on a "staggered" grid, where the velocity components are calculated at different points with respect to those where the volume fraction $a$ is calculated. In this 
paper, following the methodology of Morinishi et al. [4] and Vasilyev [5], in the staggered grid the differential terms are approximated by means of a conservative fourth-order accurate numerical scheme. The generic point of the curvilinear grid is identified by the coordinates $\xi_{i j k}^{1}, \xi_{i j k}^{2}, \xi_{i j k}^{3}$. By considering that in the computational domain the spatial step is constant and equal to one, the finite difference operator with stencil $\alpha$ acting on a generic function $\varphi$ with respect to $\xi^{1}$, can be defined as:

$$
\left.\frac{\delta_{\alpha} \varphi}{\delta_{\alpha} \xi^{1}}\right|_{\xi_{i j k}^{1}, \xi_{i j k}^{2}, \xi_{i j k}^{3}} \equiv \frac{\varphi\left(\xi_{i j k}^{1}+\alpha / 2, \xi_{i j k}^{2}, \xi_{i j k}^{3}\right)-\varphi\left(\xi_{i j k}^{1}-\alpha / 2, \xi_{i j k}^{2}, \xi_{i j k}^{3}\right)}{\alpha} .
$$

We also define an interpolation operator with stencil $\alpha$ acting on $\varphi$ in the $\xi^{1}$ direction as

$$
\left.\bar{\varphi}^{\alpha \xi^{1}}\right|_{\xi_{i j k}^{1}, \xi_{i j k}^{2}, \xi_{i j k}^{3}} \equiv \frac{\varphi\left(\xi_{i j k}^{1}+\alpha / 2, \xi_{i j k}^{2}, \xi_{i j k}^{3}\right)+\varphi\left(\xi_{i j k}^{1}-\alpha / 2, \xi_{i j k}^{2}, \xi_{i j k}^{3}\right)}{2}
$$

The combination of the discrete operator defined by Eq. (13), with different values of $\alpha$, can be used to make a higher order approximation of the first derivative of $\varphi$ with respect to $\xi^{1}$. The combination of the discrete operator defined by Eq. (14), with different values of $\alpha$, can be used to make a higher order approximation of the function value of $\varphi$. In this paper the fourth order accurate approximation of the firs derivative of $\varphi$ with respect to $\xi^{1}$ is obtained by means of Eq. (13), once with $\alpha$ equal to 1 and once with $\alpha$ equal to 3, and combining the resulting discrete operators as follows:

$$
\begin{array}{r}
\left.\frac{\delta \varphi}{\delta \xi^{1}}\right|_{\xi_{i j k}^{1}, \xi_{i j k}^{2}, \xi_{i j k}^{3}}=\left.\frac{9}{8} \frac{\delta_{1} \varphi}{\delta_{1} \xi^{1}}\right|_{\xi_{i j k}^{1}, \xi_{i j k}^{2}, \xi_{i j k}^{3}}-\left.\frac{1}{8} \frac{\delta_{3} \varphi}{\delta_{3} \xi^{1}}\right|_{\xi_{i j k}^{1}, \xi_{i j k}^{2}, \xi_{i j k}^{3}}= \\
\frac{9}{8}\left(\frac{\varphi\left(\xi_{i j k}^{1}+1 / 2, \xi_{i j k}^{2}, \xi_{i j k}^{3}\right)-\varphi\left(\xi_{i j k}^{1}-1 / 2, \xi_{i j k}^{2}, \xi_{i j k}^{3}\right)}{1}\right) \\
-\frac{1}{8}\left(\frac{\varphi\left(\xi_{i j k}^{1}+3 / 2, \xi_{i j k}^{2}, \xi_{i j k}^{3}\right)-\varphi\left(\xi_{i j k}^{1}-3 / 2, \xi_{i j k}^{2}, \xi_{i j k}^{3}\right)}{3}\right)
\end{array}
$$

\section{Results and discussion}

The validation of the proposed numerical model is performed by comparing the numerical results with the experimental data produced by Hosseini et al. [1].

In particular, Fig. 1(a) shows the velocity and concentration fields that are produced by the numerical simulation (Simulation 1) in a domain representing the rectangular tank (whose dimensions are $12 \mathrm{~m}, 1,5 \mathrm{~m}$ and $0,75 \mathrm{~m}$ ) used by (...) to produce the experimental data.

The above-mentioned velocity and concentration fields (shown in Fig. 1) were obtained by assuming the solid particle specific gravity $\tilde{\rho}_{s} / \rho=2.65$, and a 
solid particle diameter $d=0.02 \mathrm{~mm}$; the bottom slope, the inflow concentration, the liquid flow rate and velocity were assumed to be, respectively, equal to $3 \%$, $0.005 \mathrm{~kg} / \mathrm{dm}^{3}, 8 \mathrm{l} / \mathrm{min}$ and $13 \mathrm{~cm} / \mathrm{sec}$; Fig. 1(b) shows the detail of the velocity and concentration field obtained at an instant of the simulation corresponding to about 275 seconds from the moment at which water and solid material were released into the tank of fresh water.
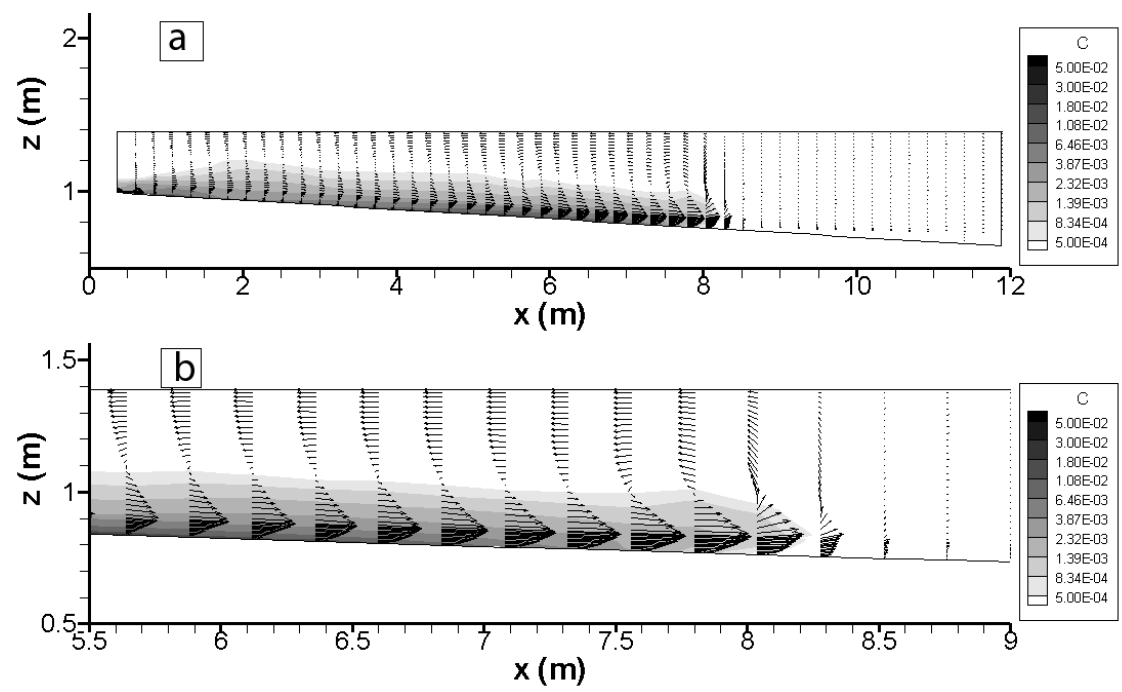

Figure 1: Numerical simulation of the turbidity current inside the computational domain that reproduces the laboratory apparatus: a) vertical section of the velocity and concentration fields obtained by means of the proposed simulation model; b) detail of the velocity and concentration fields. Simulation 1.

Fig 2(a) shows the comparison between the mean downstream velocity values produced by the numerical simulation and the corresponding values obtained by experimental data, while Fig. 2(b) shows the mean concentration profile obtained by the numerical simulation and the profile obtained by experimental data [1] (both are relative to the case shown in Fig. 1). The velocity maximum ( Um ), as the characteristic velocity, and the height of the velocity maximum $(\mathrm{Hm})$ and the depth-averaged thickness of the current $(H)$, as length scales, were used to obtain the non-dimensional form of the velocity profiles [6]; $\mathrm{Cm}$ is the mean concentration at the height of the velocity maximum.

Figs 3(a) and 3(b) show the comparison between the mean velocity and the mean concentration profiles produced by the numerical simulation (Simulation 2) and those obtained from the experimental data; in this case the bottom slope is equal to $3 \%$, the inflow concentration is equal to $0.01 \mathrm{~kg} / \mathrm{dm}^{3}$, the inflow liquid flow rate and velocity are, respectively, equal to $12 \mathrm{l} / \mathrm{min}$ and $20 \mathrm{~cm} / \mathrm{sec}$. In 

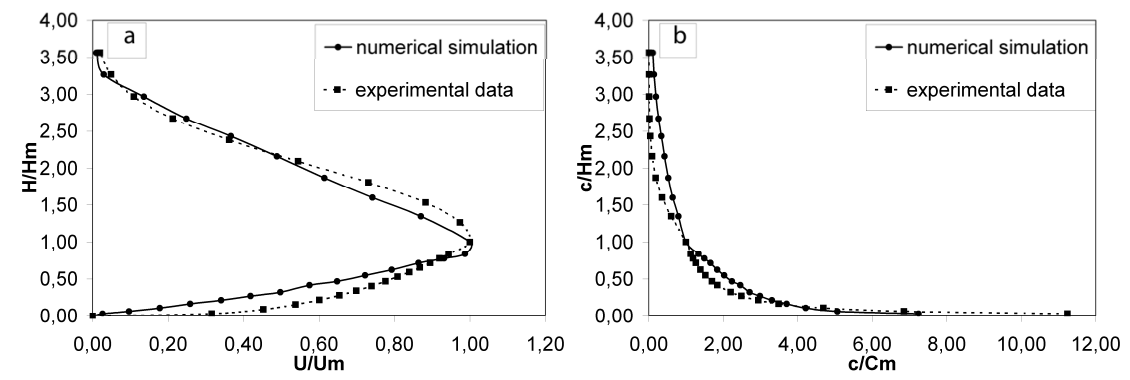

Figure 2: Comparison between the numerical simulation and the experimental data: a) dimensionless velocity profiles; b) dimensionless concentration profiles. Simulation 1.
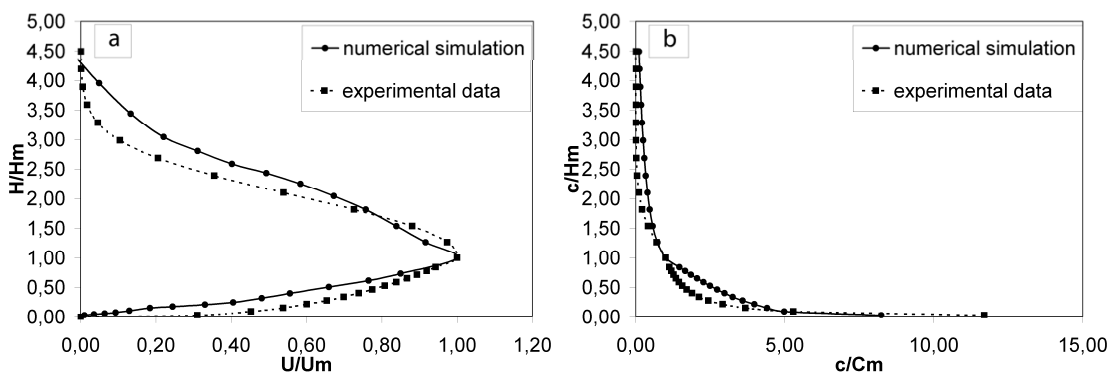

Figure 3: Comparison between the numerical simulation and the experimental data: a) dimensionless velocity profiles; b) dimensionless concentration profiles. Simulation 2 .
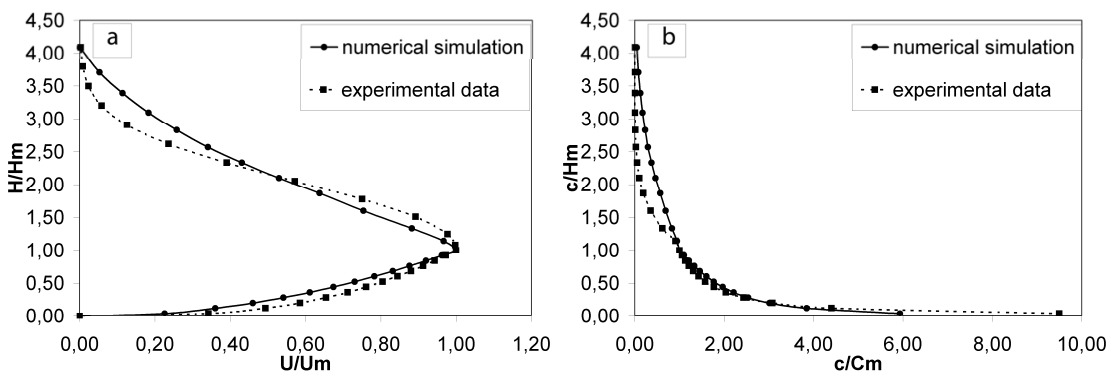

Figure 4: Comparison between the numerical simulation and the experimental data: a) dimensionless velocity profiles; b) dimensionless concentration profiles. Simulation 3 . 
Figs 4(a) and 4(b) compare the mean velocity and the mean concentration profiles obtained by the numerical simulation (Simulation 3) and those obtained from the experimental data; in this case the bottom slope is equal to $2 \%$, the inflow concentration, the inflow liquid flow rate and the inflow liquid velocity are, respectively, $0.005 \mathrm{~kg} / \mathrm{dm}^{3}, 10 \mathrm{l} / \mathrm{min}$ and $16 \mathrm{~cm} / \mathrm{sec}$. From the figures the agreement between the numerical results and the experimental data can clearly be seen.
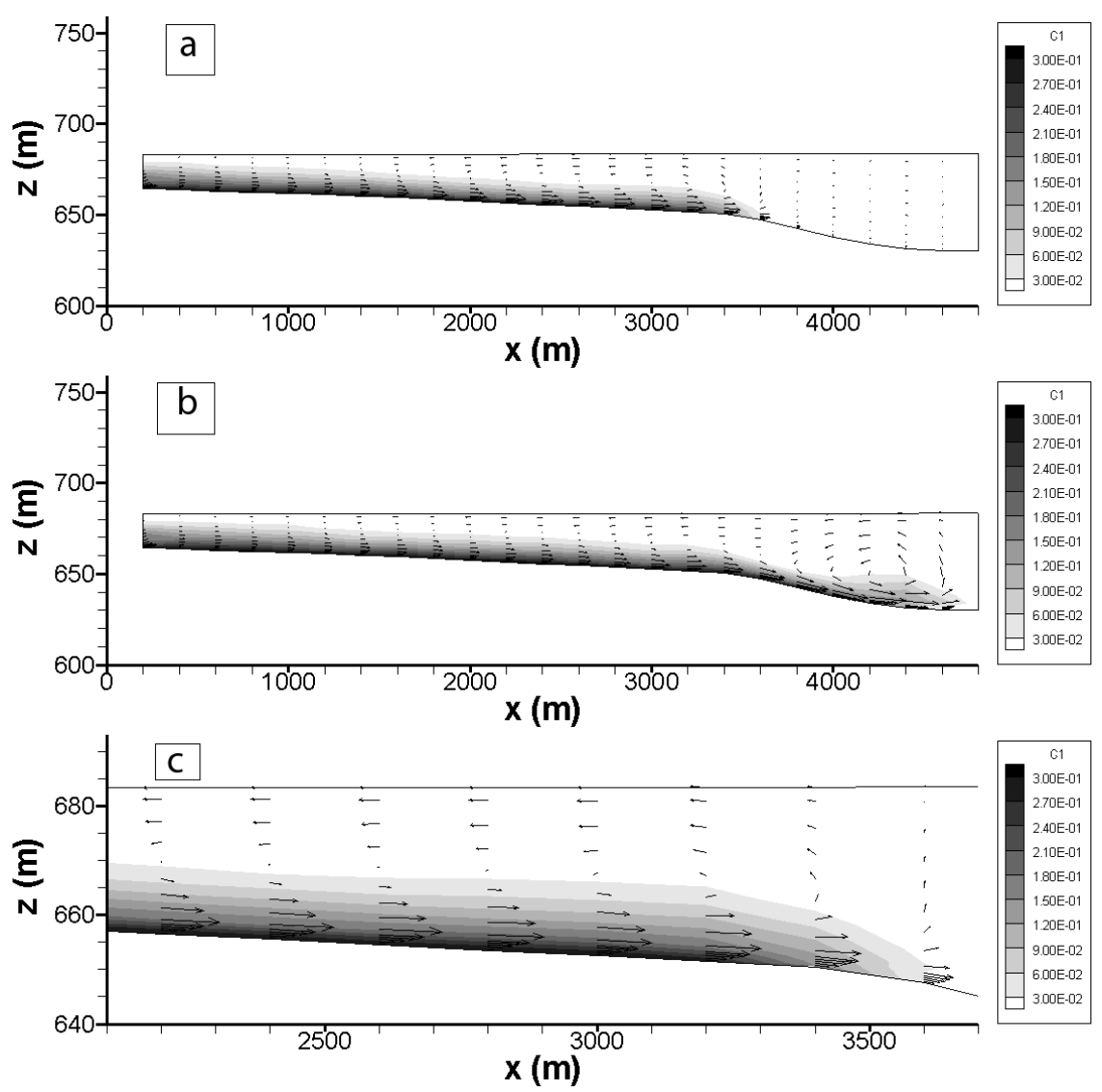

Figure 5: Numerical simulation of the turbidity current in the Pieve di Cadore reservoir: a) vertical section of the velocity and concentration fields relative to about 90 minutes from the beginning of the arrival of the flood; b) vertical section of the velocity and concentration fields relative to about 100 minutes from the beginning of the arrival of the flood; c) detail of the velocity and concentration fields after about 90 minutes from the beginning of the arrival of the flood. Simulation 4 . 
The simulation model presented in the previous sections was applied to the case study of the Pieve di Cadore reservoir, in conditions of a full reservoir and liquid and solid inflow discharge in the reservoir that can be associated with flood events with a return period of less than ten years. In the simulations carried out, conditions were reproduced of the opening of the bottom outlet such as to ensure the complete release of the flood through the bottom outlet itself, without significant changes of water level in the reservoir.

Fig. 5 shows the velocity and concentration fields (Simulation 4) produced by a flood characterized by the following values of the principal hydraulics quantities: specific gravity and diameter of the solid particles carried by the tributary, $\tilde{\rho}_{s} / \rho=2.65$, and $d=0.1 \mathrm{~mm}$; average concentration of suspended solids over the inlet cross section of the reservoir, $C=5 \mathrm{~g} / 1$; flood peak value of the tributary, $Q=100 \mathrm{~m}^{3} / \mathrm{s}$. These values are characteristic of flood events with a return period equal to two years. The above figures show that, in such conditions, a turbidity current is generated and the present bottom slopes of the reservoir are high enough to let the turbidity current reach the bottom outlet.
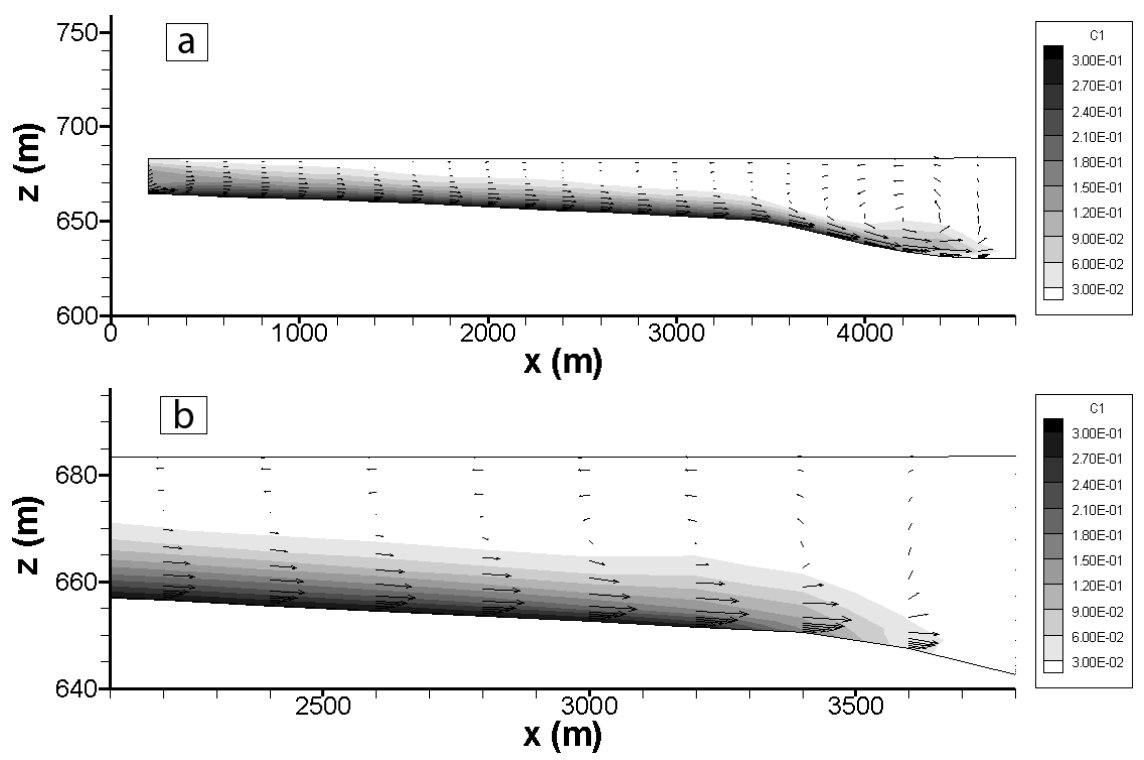

Figure 6: Numerical simulation of the turbidity current in the Pieve di Cadore reservoir: a) vertical section of the velocity and concentration fields relative to about 85 minutes from the beginning of the arrival of the flood; b) detail of the velocity and concentration fields after about 75 minutes from the beginning of the arrival of the flood. Simulation 5. 
Figs 6 and 7 show the velocity and concentration fields (Simulations 5 and 6 , respectively) produced by a flood event characterized by a flood peak value equal to the maximum value that is able to flow through the bottom outlet when the reservoir is full $\left(Q=180 \mathrm{~m}^{3} / \mathrm{s}\right)$ and by an average concentration value of suspended solids over the inlet cross-section of the reservoir equal to $C=15 \mathrm{~g} / 1$. These values are less than the characteristic ones for flood events with a return period of ten years (which are, respectively, $Q=250 \mathrm{~m}^{3} / \mathrm{s}$ and $C=20 \mathrm{~g} / 1$ ). The results shown in Fig 6 were obtained by assuming a diameter of solid particles carried by the tributary equal to $d=0.1 \mathrm{~mm}$, while the results shown in Fig 10 were obtained by assuming a diameter of solid particles equal to $d=0.3 \mathrm{~mm}$.

From Figs 6 and 7 it can be seen that, in the simulated hydraulic conditions, a turbidity current is generated that is able to carry ever coarser solid material to the bottom outlets. The results of the numerical simulations show that, in the Pieve di Cadore reservoir, for inflow liquid and solid discharge values comparable with the flood values with a return period of less than ten years, the inlet concentration values in the reservoir are such as to allow the formation of turbidity currents. The present bottom slopes of the reservoir are high enough so as to let the turbidity current reach the bottom outlet.
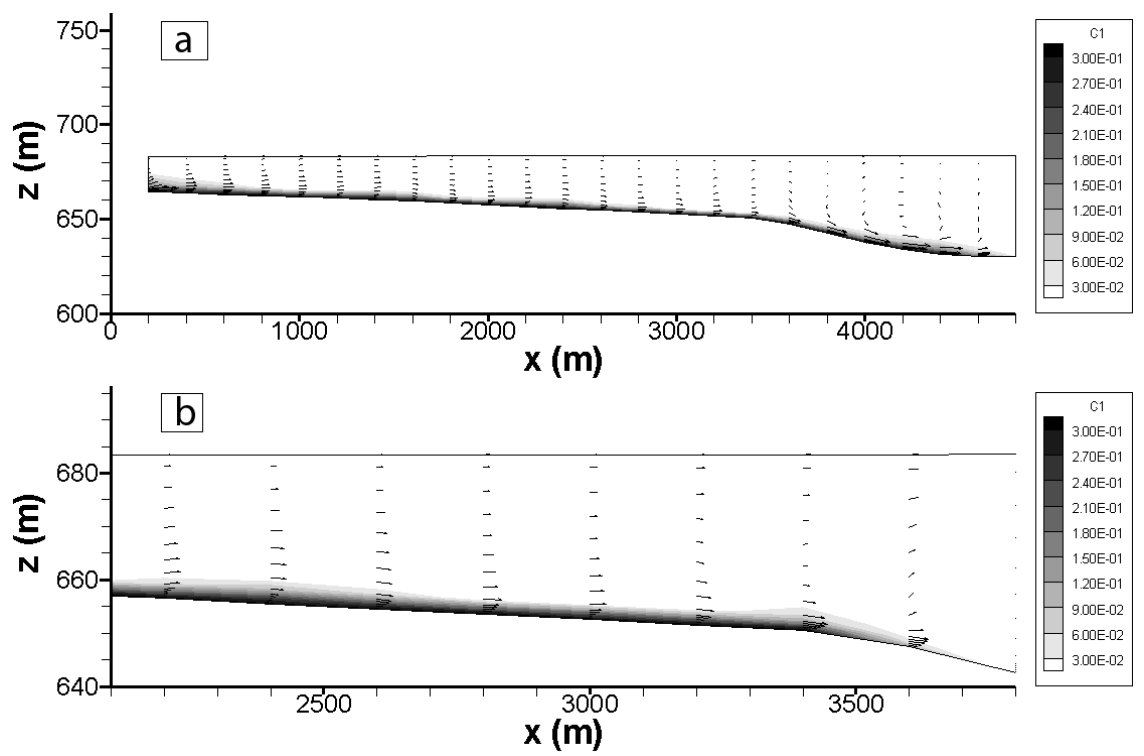

Figure 7: Numerical simulation of the turbidity current in the Pieve di Cadore reservoir: a) vertical section of the velocity and concentration fields relative to about 100 minutes from the beginning of the arrival of the flood; $b$ ) detail of the velocity and concentration fields after about 87 minutes from the beginning of the arrival of the flood. Simulation 6. 


\section{Conclusions}

In this paper a model for the simulation of the turbidity currents is proposed; the model is based on the numerical integration of the two phase flow motion equations. These equations are integrated on a three-dimensional generalised curvilinear grid and are directly expressed in contravariant formulation. The numerical model is validated by the comparison between the numerical results and experimental data and is applied to the reservoir of Pieve di Cadore (Italy). The results of the numerical simulations show that, in the Pieve di Cadore reservoir, the inlet liquid and solid discharge with a return period of less than ten years produce concentration fields in the reservoir such as to allow the formation, near the bottom, of turbidity currents. The present bottom slopes of the reservoir are high enough to let the turbidity current reach the bottom outlet. It can be concluded that, at the Pieve di Cadore dam, it is possible to use the bottom outlets for the optimal control of the silting process in the reservoir.

\section{References}

[1] Hosseini, S.A., Shamsai, A., Ataie-Ashtiani, B., Synchronous measurements of the velocity and concentration in low density turbidity currents using an Acoustic Doppler Velocimeter. Flow Measurement and Instrumentation, 17, pp. 59-68, 2006.

[2] Thompson, J.F., Warsi, Z.U.A., Mastin, C.W., Transformation Relations (Chapter 4). Numerical Grid Generation, ed. North-Holland: New York, Amsterdam and New York, pp. 94-135, 1985.

[3] Rutherford, A., Tensors (Chapter 7). Vectors, Tensors and the Basic Equations of Fluid Mechanics, ed. Dover Publications, Inc, pp. 135-175, 1989.

[4] Morinishi, Y., Lund, T.S., Vasiliyev. O.V., Moin, P., Fully Conservative Higher Order Finite Difference Schemes for Incompressible Flow. Journal of Computational Physics, 143, pp. 90-124, 1998.

[5] Vasiliyev. O.V., Higher Order Finite Difference Schemes on Non-uniform Meshes with Good Conservation Properties. Journal of Computational Physics, 157, pp. 746-761, 2000.

[6] Altinakar, M.S., Graf, W.H., Hopfinger, E.J., Flow structure in turbidity currents. Journal of Hydraulic Research, 34, pp. 713-718, 1996. 